\title{
PS-Regular Sets in Topology and Generalized Topology
}

\author{
Ankit Gupta $^{1}$ and Ratna Dev Sarma ${ }^{2}$ \\ ${ }^{1}$ Department of Mathematics, University of Delhi, Delhi 110007, India \\ ${ }^{2}$ Department of Mathematics, Rajdhani College, University of Delhi, Delhi 110015, India
}

Correspondence should be addressed to Ratna Dev Sarma; ratna_sarma@yahoo.com

Received 23 May 2014; Revised 25 August 2014; Accepted 26 August 2014; Published 14 September 2014

Academic Editor: Peter R. Massopust

Copyright (C) 2014 A. Gupta and R. D. Sarma. This is an open access article distributed under the Creative Commons Attribution License, which permits unrestricted use, distribution, and reproduction in any medium, provided the original work is properly cited.

We define and study a new class of regular sets called PS-regular sets. Properties of these sets are investigated for topological spaces and generalized topological spaces. Decompositions of regular open sets and regular closed sets are provided using PS-regular sets. Semiconnectedness is characterized by using PS-regular sets. PS-continuity and almost PS-continuity are introduced and investigated.

\section{Introduction}

In general topology, repeated applications of interior and closure operators give rise to several different new classes of sets. Some of them are generalized form of open sets while few others are the so-called regular sets. These classes are found to have applications not only in mathematics but even in diverse fields outside the realm of mathematics [1-3].

Due to this, investigations of these sets have gained momentum in the recent days. Császár has already provided an umbrella study for generalized open sets in his latest papers [4-7]. In this paper, we introduce and study a new class of sets, called PS-regular sets, using semi-interior and semiclosure operators. Initially, we define them for a broader class, that is, for generalized topological spaces and discuss their various properties. Interrelationship of $P S$-regular sets with other existing classes such as semiopen sets, regular open sets, $t$-sets, $\alpha^{*}$-sets, $B$-sets, and $C$-sets has been studied. A characterization of semiconnectedness is also provided using $P S$-regular sets. Moreover, $\xi$-regular sets, where $\xi \in$ $\{\alpha, \beta, \pi, \sigma\}$, of a generalized topological space are studied using PS-regular sets. In the last two sections, PS-regularity is studied in the domain of general topological spaces. Here several decompositions of regular open sets and regular closed sets are provided using $P S$-regular sets. In the last section, $P S$-continuity and almost $P S$-continuity are defined and interrelationship of almost PS-continuity with other existing mappings such as $R$-map, graph mapping, almost precontinuity, and almost $\alpha$-continuity is investigated.

\section{Preliminaries}

First we recall some definitions and results to be used in the paper.

Definition 1 (see [6]). Let $X$ be a nonempty set. A collection $\lambda$ of subsets of $X$ is called a generalized topology (in brief, GT) on $X$ if it is closed under arbitrary unions. The ordered pair $(X, \lambda)$ is called generalized topological space (in brief, GTS).

Since an empty union amounts to the empty set, $\emptyset$ always belongs to $\lambda$. However, $X$ need not be a member of $\lambda$. The members of $\lambda$ are called $\lambda$-open while the complements of $\lambda$-open sets are called $\lambda$-closed. The largest $\lambda$-open set contained in a set $A$ is called the interior of $A$ and is denoted by $i_{\lambda}(A)$, whereas the smallest $\lambda$-closed set containing $A$ is called the closure of $A$ and is denoted by $c_{\lambda}(A)$. For $A, B \subseteq X$, we have, $A \subseteq c_{\lambda}(A), c_{\lambda}\left(c_{\lambda}(A)\right)=c_{\lambda}(A)$ and whenever $A \subseteq B$, we have $c_{\lambda}(A) \subseteq c_{\lambda}(B)$. These properties will be used in the text without any further mention.

Remark 2. Although Császár provided the definition of a generalized topology, similar notions existed prior to Császár's work also. Peleg [8] in 1984 defined a similar 
structure which he named "semitopology" on $X$. The corresponding "semitopological closure" is found to be monotone, enlarging, and idempotent. A subfamily $\mathscr{C}$ of the power set of $X$ which is closed under nonempty intersection has been studied in the literature under the name of "intersection structure" [9]; the corresponding topped intersection structure is known as closure system.

Definition 3. Let $(X, \lambda)$ be a GTS. Then a subset $A$ of $X$ is called

(i) $\lambda$-semiopen [7] if $A \subseteq c_{\lambda} i_{\lambda}(A)$;

(ii) $\lambda$ - $\alpha$-open [7] if $A \subseteq i_{\lambda} c_{\lambda} i_{\lambda}(A)$;

(iii) $\lambda$-preopen [7] if $A \subseteq i_{\lambda} c_{\lambda}(A)$;

(iv) $\lambda$ - $\beta$-open [7] if $A \subseteq c_{\lambda} i_{\lambda} c_{\lambda}(A)$;

(v) $\alpha^{*}$-set [10] if $i_{\lambda}(A)=i_{\lambda}\left(c_{\lambda}\left(i_{\lambda}(A)\right)\right)$;

(vi) regular open (resp., regular closed) [11] if $A=i_{\lambda} c_{\lambda}(A)$ (resp., $A=c_{\lambda} i_{\lambda}(A)$ ).

The complement of a $\lambda$-semiopen (resp., $\lambda$ - $\alpha$-open, $\lambda$ preopen, and $\lambda$ - $\beta$-open) set is called $\lambda$-semiclosed (resp., $\lambda$ $\alpha$-closed, $\lambda$-preclosed, and $\lambda$ - $\beta$-closed).

The families of regular open, regular closed, and $\alpha^{*}$-sets on $(X, \lambda)$ are denoted by $R O(X, \lambda), R C(X, \lambda)$ and $\alpha^{*}(X, \lambda)$, respectively.

The intersection of all $\lambda$-semiclosed sets containing a set $A$ is called the semiclosure of $A$ and is denoted by $c_{\sigma}(A)$. Dually, the semi-interior of $A$ is defined to be the union of all $\lambda$-semiopen sets contained in $A$ and is denoted by $i_{\sigma}(A)$.

Theorem 4 (see [5]). In a GTS $(X, \lambda)$ with $A \subseteq X$, one has

(i) $c_{\sigma}(A)=A \cup i_{\lambda} c_{\lambda}(A)$,

(ii) $i_{\sigma}(A)=A \cap c_{\lambda} i_{\lambda}(A)$,

where $i_{\sigma}$ and $c_{\sigma}$ denote the semi-interior and semiclosure of $A$, respectively.

\section{3. $P S$-Regular Sets in Generalized Topological Spaces}

Although the following discussion is provided for generalized topological space, it is also valid for topological spaces as every topological space is a generalized topological space as well.

Definition 5. A subset $A \subseteq X$ of a generalized topological space $(X, \lambda)$ is said to be $P S$-regular if $A=i_{\sigma}\left(c_{\sigma}(A)\right)$.

The class of all PS-regular sets in $(X, \lambda)$ is denoted by $\operatorname{PS}(X, \lambda)$.

Lemma 6. For a generalized topological space $(X, \lambda), \emptyset$ and $X$ both are PS-regular sets.

Proof. Let $(X, \lambda)$ be a GTS. Then $c_{\sigma}(X)=X \cup i_{\lambda} c_{\lambda}(X)=X$. Now, consider $i_{\sigma}(X)=X \cap c_{\lambda} i_{\lambda}(X)$. If $i_{\lambda}(X)=X$, then $i_{\sigma}(X)=X$ and we are done. If not, then let $i_{\lambda}(X)=U$, the largest $\lambda$-open set in $X$. If $c_{\lambda}(U)=X$ then again $i_{\sigma}(X)=X$ and $X$ is $P S$-regular. Let, if possible, $c_{\lambda}(U) \neq X$, and then $X \backslash c_{\lambda}(U)$ is a $\lambda$-open set in $X$ and hence $U \cup\left(X \backslash c_{\lambda}(U)\right)$ is again a $\lambda$-open set containing $U$, which leads to a contradiction. Hence $X$ is $P S$-regular. Similarly, it can be shown that $\emptyset$ is $P S$ regular.

Thus one can say that the family of PS-regular sets $P S(X, \lambda)$ forms an $m$-structure [12]. However, the family $\operatorname{PS}(X, \lambda)$ is not closed under finite union as well as finite intersection.

We have the following example.

Example 7. Let $X=\mathbb{R}$ be the set of all real numbers with the usual topology $\lambda$. Take $A=(1,3]$ and $B=[3,5)$; both are $P S$ regular sets. But $A \cap B=\{3\}$ is not a $P S$-regular set because $i_{\sigma}\left(c_{\sigma}\{3\}\right)=\phi \neq A \cap B$.

Similarly, take $C=[1,2)$ and $D=(2,3]$; both are $P S$ regular sets. But $C \cup D=[1,2) \cup(2,3]$ is not a $P S$-regular set because $i_{\sigma}\left(c_{\sigma}(C \cup D)\right)=[1,3] \neq C \cup D$.

Theorem 8. Every PS-regular set is $\lambda$-semiopen.

Proof. Let $A$ be a PS-regular set. Then $A=i_{\sigma}\left(c_{\sigma}(A)\right)$. Therefore $i_{\sigma}(A)=i_{\sigma}\left(i_{\sigma}\left(c_{\sigma}(A)\right)=i_{\sigma}\left(c_{\sigma}(A)\right)=A\right.$. Hence $A$ is $\lambda$-semiopen.

But converse of this result is not true in general. We have the following example.

Example 9. Let $X=\{a, b, c, d\}$ with $G T \lambda=\{\emptyset,\{b, c\}$, $\{a, b, c\}\}$. Then $A=\{b, c\}$ is a $\lambda$-semiopen set because $A$ is $\lambda$ open. But $A$ is not $P S$-regular because $i_{\sigma}\left(c_{\sigma}(A)\right)=\{a, b, c\} \neq$ A.

Remark 10. The notions of $P S$-regular sets and $\lambda$-open sets are independent of each other. Similarly, PS-regular sets are different from $\lambda$ - $\alpha$-open, $\lambda$-preopen, and $\lambda$ - $\beta$-open sets as well.

In Example 9 mentioned above, it is clear that $A=\{b, c\}$ is a $\lambda$-open set; thus it is $\lambda$ - $\alpha$-open, $\lambda$-preopen, and $\lambda$ - $\beta$-open. But $A$ is not $P S$-regular.

Example 11. Let $X=\mathbb{R}$ be the set of all real numbers with its usual topology $\lambda$. Then $A=(5,6) \cap \mathbb{Q}$ is a $\lambda$-preopen set in $(X, \lambda)$ as $A \subseteq i_{\lambda}\left(c_{\lambda}(A)\right)=(5,6)$. But $A$ is not $P S$-regular because $i_{\sigma}\left(c_{\sigma}(A)\right)=(5,6) \neq A$.

Thus PS-regular sets are independent of $\lambda$-preopen sets.

Example 12. Let $X=\{a, b, c, d\}$ with $G T \lambda=\{\emptyset,\{a\},\{b, c\}$, $\{a, b, c\}\}$. Then $A=\{a, d\}$ is a $P S$-regular set. But $A$ is neither $\lambda$-open nor $\lambda$-preopen or $\lambda$ - $\alpha$-open in $(X, \lambda)$ because $i_{\lambda}\left(c_{\lambda}(A)\right)=\{a\}$ and $i_{\lambda}\left(c_{\lambda}\left(i_{\lambda}(A)\right)=\{a\}\right.$.

Hence from the above examples, it is clear that $P S$-regular sets are independent of $\lambda$-open sets, $\lambda$-preopen sets, and $\lambda$ - $\alpha$ open sets as well. 
Theorem 13. Let $(X, \lambda)$ be a generalized topological space. Then a subset $A \subseteq X$ is PS-regular if it is both $\lambda$-semiopen and $\lambda$-semiclosed.

Proof. Let $A \subseteq X$ be $\lambda$-semiopen as well as $\lambda$-semiclosed. Then $A=c_{\sigma}(A)$ and $A=i_{\sigma}(A)$. Therefore $i_{\sigma}\left(c_{\sigma}(A)\right)=A$. Hence $A$ is $P S$-regular.

Theorem 14. Every regular open set is PS-regular.

Proof. Let $A$ be a regular open set. Therefore $A$ is $\lambda$-open and hence $A$ is $\lambda$-semiopen. Again as $A$ is regular open, we have $A=i_{\lambda}\left(c_{\lambda}(A)\right)$. Thus $c_{\sigma}(A)=A \cup i_{\lambda}\left(c_{\lambda}(A)\right)=A$. Hence $A$ is $\lambda$-semiclosed. Therefore by Theorem 13, it follows that $A$ is $P S$-regular.

But the converse of the above result is not true in general. We have the following example.

Example 15. In Example 12, $A=\{a, d\}$ is $P S$-regular. But $A$ is not regular open as $i_{\lambda}\left(c_{\lambda}(A)\right)=\{a\} \neq A$.

Theorem 16. Every regular closed set is PS-regular.

Proof. Let $A \subseteq X$ be regular closed. Therefore $A$ is $\lambda$-closed and $\lambda$-semiclosed. As $A$ is regular closed, $A=c_{\lambda}\left(i_{\lambda}(A)\right)$ and hence $A \subseteq c_{\lambda}\left(i_{\lambda}(A)\right)$. Thus $A$ is both $\lambda$-semiopen and $\lambda$ semiclosed. Hence $A$ is $P S$-regular in $(X, \lambda)$.

But the converse of the above theorem is not true in general. We have the following example.

Example 17. Let $X=\mathbb{R}$ be the set of all real numbers with the usual topology $\lambda$. Then $A=[5,6)$ is not a regular closed set. But it is $P S$-regular.

In this section, we provide two interesting characterizations of $P S$-regular sets.

Theorem 18. Let $(X, \lambda)$ be a generalized topological space. Then a subset $A \subseteq X$ is PS-regular if and only if $A=[A \cap$ $\left.c_{\lambda}\left(i_{\lambda}\left(c_{\lambda}(A)\right)\right)\right] \cup i_{\lambda}\left(c_{\lambda}(A)\right)$.

Proof. For a generalized topological space, we know that

$$
i_{\sigma}(A)=A \cap c_{\lambda}\left(i_{\lambda}(A)\right), \quad c_{\sigma}(A)=A \cup i_{\lambda}\left(c_{\lambda}(A)\right) .
$$

Therefore, we have

$$
\begin{aligned}
i_{\sigma}\left(c_{\sigma}(A)\right) & =c_{\sigma}(A) \cap c_{\lambda}\left(i_{\lambda}\left(c_{\sigma}(A)\right)\right) \\
& =c_{\sigma}(A) \cap c_{\lambda}\left(i_{\lambda}\left[A \cup i_{\lambda}\left(c_{\lambda}(A)\right)\right]\right) .
\end{aligned}
$$

Consider $i_{\lambda}\left[A \cup i_{\lambda}\left(c_{\lambda}(A)\right)\right] \subseteq i_{\lambda}(A) \cup i_{\lambda}\left(c_{\lambda}(A)\right)=i_{\lambda}\left(c_{\lambda}(A)\right)$. But $i_{\lambda}\left(c_{\lambda}(A)\right) \subseteq A \cup i_{\lambda}\left(c_{\lambda}(A)\right)$; thus $i_{\lambda}\left(i_{\lambda}\left(c_{\lambda}(A)\right)\right) \subseteq i_{\lambda}[A \cup$ $\left.i_{\lambda}\left(c_{\lambda}(A)\right)\right]$. Therefore $i_{\lambda}\left[A \cup i_{\lambda}\left(c_{\lambda}(A)\right)\right]=i_{\lambda}\left(c_{\lambda}(A)\right)$. Thus, $i_{\sigma}\left(c_{\sigma}(A)\right)=c_{\sigma}(A) \cap c_{\lambda}\left(i_{\lambda}\left(c_{\sigma}(A)\right)\right)=c_{\sigma}(A) \cap c_{\lambda}\left(i_{\lambda}(A \cup\right.$ $\left.\left.i_{\lambda}\left(c_{\lambda}(A)\right)\right)\right)=\left[A \cup i_{\lambda} c_{\lambda}(A)\right] \cap c_{\lambda}\left(i_{\lambda}\left(c_{\lambda}(A)\right)\right)=[A \cap$ $\left.c_{\lambda}\left(i_{\lambda}\left(c_{\lambda}(A)\right)\right)\right] \cup i_{\lambda}\left(c_{\lambda}(A)\right)$, as $i_{\lambda}\left[A \cup i_{\lambda}\left(c_{\lambda}(A)\right)\right]=i_{\lambda}\left(c_{\lambda}(A)\right)$ (shown above). Thus $A \subseteq X$ is $P S$-regular if and only if $A=\left[A \cap c_{\lambda}\left(i_{\lambda}\left(c_{\lambda}(A)\right)\right)\right] \cup i_{\lambda}\left(c_{\lambda}(A)\right)$.

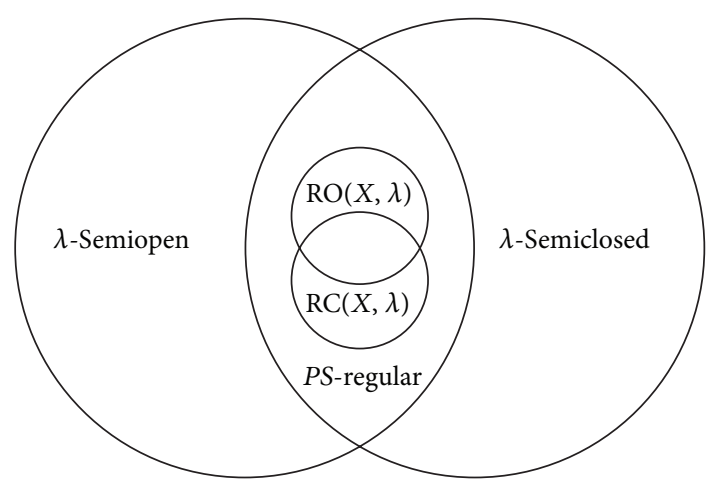

Figure 1

Corollary 19. A set A is PS-regular if and only if it is both $\lambda$ semiopen and $\lambda$-semiclosed.

Proof. From the above result, it follows that if $A$ is PS-regular set then $i_{\lambda}\left(c_{\lambda}(A)\right) \subseteq A$. Hence $A$ is $\lambda$-semiclosed. Thus the result follows in the light of Theorems 8 and 13 .

Corollary 20. If a set A is PS-regular then its complement is also PS-regular.

The above implications may be represented diagrammatically in Figure 1.

We complete this section by providing few interesting decompositions and results using PS-regular sets.

Definition 21 (see [13]). Let $(X, \lambda)$ be a generalized topological space and $A \subseteq X$. $A$ is said to be $\alpha$-regular (resp., $\sigma$-regular, $\pi$-regular, and $\beta$-regular) if $A=i_{\lambda} c_{\lambda} i_{\lambda}(A)$ (resp., $A=c_{\lambda} i_{\lambda}(A), A=i_{\lambda} c_{\lambda}(A)$, and $\left.A=c_{\lambda} i_{\lambda} c_{\lambda}(A)\right)$.

The following theorems provide the relationships between $\xi$-regular sets where $\xi \in\{\alpha, \pi, \sigma, \beta\}$ and PS-regular sets. Here it may be mentioned that $\sigma$-regular sets and $\pi$-regular sets are nothing but regular open sets and regular closed sets defined in Definition 3.

Theorem 22. Let $(X, \lambda)$ be a GTS and $A \subseteq X$. Then every $\xi$ regular set, where $\xi \in\{\alpha, \pi, \sigma, \beta\}$, is PS-regular.

Proof. Let $A$ be $\pi$-regular. Then $A=i_{\lambda} c_{\lambda}(A)$. Hence by Theorem 14, it is $P S$-regular. Similarly if $A$ is $\sigma$-regular, then $A=c_{\lambda} i_{\lambda}(A)$. Hence by Theorem 16, $A$ is $P S$-regular as well. Further, we know that a set is $\pi$-regular if and only if it is an $\alpha$ regular [13] and a set is $\sigma$-regular if and only if it is $\beta$-regular [13]. Therefore every $\xi$-regular set, where $\xi \in\{\sigma, \pi, \alpha, \beta\}$, is PS-regular.

Theorem 23. Let $(X, \lambda)$ be a GTS and $A \subseteq X$. Then the following hold.

(i) $A$ is $\sigma$-regular if and only if $A$ is PS-regular and $\lambda$ closed if and only if $A$ is PS-regular and $\pi$-closed.

(ii) $A$ is $\beta$-regular if and only if $A$ is PS-regular and $\lambda$ closed if and only if $A$ is PS-regular and $\alpha$-closed. 
Proof. (i) If $A$ is $\sigma$-regular then it is clear that $A$ is PSregular and $\lambda$-closed which implies that $A$ is $\pi$-closed as well. Suppose $A$ is $P S$-regular and $\pi$-closed. Then $A=i_{\sigma}\left(c_{\sigma}(A)\right)$; that is, $A \subseteq c_{\lambda} i_{\lambda}(A)$ and $c_{\lambda} i_{\lambda}(A) \subseteq A$. Hence $A=c_{\lambda} i_{\lambda}(A)$ which implies that $A$ is $\sigma$-regular.

(ii) It is similar to (i).

Definition 24. A subset $A$ of a generalized topological space $(X, \lambda)$ is called $\pi g s \lambda$-closed $[14]$ if $c_{\sigma}(A) \subseteq U$ whenever $A \subseteq U$ and $U$ is $\pi \lambda$-open in $X$, and the finite unions of regular open sets are said to be $\pi \lambda$-open.

Theorem 25. Let $(X, \lambda)$ be a GTS and $A \subseteq X$. Then the following hold.

(i) Every PS-regular set is $\pi g s \lambda$-closed set.

(ii) $A$ is $\pi$-regular if and only if $A$ is $\pi \lambda$-open and PSregular.

Proof. (i) Let $A$ be a PS-regular set. Let $U$ be any $\pi \lambda$-open set in $X$ containing $A, A \subseteq U$. Since $A$ is $P S$-regular and every $P S$-regular set is $\lambda$-semiclosed in $X$, therefore, $c_{\sigma}(A) \subseteq U$. Hence $A$ is $\pi g s \lambda$-closed.

(ii) If $A$ is $\pi$-regular then clearly $A$ is $\pi \lambda$-open and $P S$ regular set. Conversely, let $A$ be $\pi \lambda$-open and $P S$-regular set. Hence $A$ is $\lambda$-semiclosed; that is, $c_{\sigma}(A) \subseteq A$; that is, $i_{\lambda} c_{\lambda}(A) \subseteq$ $A$. Since $A$ is $\lambda$-open set, therefore $A$ is $\lambda$-preopen and hence $A \subseteq i_{\lambda} c_{\lambda}(A)$. Thus $A=i_{\lambda} c_{\lambda}(A)$. Hence $A$ is a $\pi$-regular set.

An application of $P S$-regular set is the characterization of semiconnectedness [15].

Definition 26. A generalized topological space $(X, \lambda)$ is said to be semidisconnected if there exist two $\lambda$-semiopen sets $A$ and $B$ such that $X=A \cup B$ and $A \cap B=\emptyset$; otherwise it is called semiconnected.

Theorem 27. A generalized topological space is semiconnected if and only if it does not contain any proper PS-regular set.

Proof. Suppose $A$ is a proper $P S$-regular set in $(X, \lambda)$. Then $B=X \backslash A$ is also a $P S$-regular set. Therefore $A$ and $B$ both are $\lambda$-semiopen sets such that $A \cup B=X$ and $A \cap B=\emptyset$. Hence $(X, \lambda)$ is semidisconnected.

Conversely, let $(X, \lambda)$ be a semidisconnected space. Then there exist two $\lambda$-semiopen sets $A$ and $B$ such that $A \cup B=X$ and $A \cap B=\emptyset$. Then $A=X \backslash B$, a $\lambda$-semiclosed set as well. Hence $A$ is $\lambda$-semiopen and $\lambda$-semiclosed both. Therefore $A$ is a proper $P S$-regular set in $X$.

Our investigations on PS-regular sets so far have been in the domain of generalized topological spaces. In the remaining part of our paper, we study interrelationships of the notion of $P S$-regularity with other existing topological notions. Hence, from now onwards, we confine our investigation to the domain of topological spaces only. Also, following the usual convention of topology, we denote interior and closure of a set $A$ by $\operatorname{int}(A)$ and $\operatorname{cl}(A)$, respectively, in our discussion. Since a topological space is also a generalized topological space, therefore all the results of this section so far are also valid for topological spaces.

\section{Some Decompositions Using PS-Regular Sets}

Apart from semiopen and semiclosed sets, there are several other important generalized forms of open sets and closed sets in topology such as $\alpha^{*}$-set, $t$-set, $B$-set, $C$-set, and $D(c, \alpha)$ set. In this section, we study PS-regular sets in the light of these sets. We also provide some interesting decompositions of regular open and regular closed sets using the notion of $P S$-regular sets.

First of all, we provide the following definitions for topological spaces.

Definition 28. Let $(X, \tau)$ be a topological space. A subset $S \subseteq$ $X$ is said to be

(i) a $t$-set $[16]$ if $\operatorname{int}(\mathrm{cl}(S))=\operatorname{int}(S)$;

(ii) a $B$-set [16] if there is an open set $U$ and a $t$-set $A$ in $X$ such that $S=U \cap A$;

(iii) a $C$-set [10] if there exists $U \in \tau$ and $A \in \alpha^{*}(X, \tau)$, such that $S=U \cap A$;

(iv) a $D(C, \alpha)$-set [17] if $\operatorname{int}(S)=S \cap \operatorname{int}(\operatorname{cl}(\operatorname{int}(S)))$.

Theorem 29. Every PS-regular set is $\alpha^{*}$-set and $t$-set.

Proof. Let $A \subseteq X$ be a PS-regular set. Then $A=[A \cap$ $\operatorname{cl}(\operatorname{int}(\operatorname{cl}(A)))] \cup \operatorname{int}(\operatorname{cl}(A))$. Therefore $\operatorname{int}(A)=\operatorname{int}[[A \cap$ $\mathrm{cl}(\operatorname{int}(\mathrm{cl}(A)))] \cup \operatorname{int}(\operatorname{cl}(A))]=\operatorname{int}[(A \cup \operatorname{int}(\operatorname{cl}(A))) \cap$ $\operatorname{cl}(\operatorname{int}(\operatorname{cl}(A)))]=\operatorname{int}[A \cup \operatorname{int}(\operatorname{cl}(A))] \cap \operatorname{int}(\operatorname{cl}(\operatorname{int}(\operatorname{cl}(A))))=$ $\operatorname{int}(\operatorname{cl}(A)) \cap \operatorname{int}(\operatorname{cl}(A))=\operatorname{int}(\operatorname{cl}(A))$ because $\operatorname{int}(A \cup$ $\operatorname{int}(\operatorname{cl}(A)))=\operatorname{int}(\mathrm{cl}(A))$.

Thus $A$ is $t$-set. Also $A$ is an $\alpha^{*}$-set as well because $\operatorname{int}(\operatorname{cl}(\operatorname{int}(A)))=\operatorname{int}(\operatorname{cl}(\operatorname{int}(\operatorname{cl}(A))))=\operatorname{int}(\operatorname{cl}(A))=$ $\operatorname{int}(A)$.

But the converse is not always true. We have the following examples.

Example 30. Let $X=\{a, b, c\}$ with topology $\tau=\{\emptyset, X,\{a\}$, $\{a, b\}\}$. Then $A=\{b\}$ is a $t$-set because $\operatorname{int}(\operatorname{cl}(A))=\emptyset=$ $\operatorname{int}(A)$. But $A$ is not $P S$-regular because $\{b\}$ is not semiopen as $\operatorname{cl}(\operatorname{int}(A))=\emptyset \nsupseteq A$.

Example 31. Let $X=\{a, b, c, d\}$ with topology $\tau=\{\emptyset$, $X,\{d\},\{b, c\},\{b, c, d\}\}$. Then $A=\{a, b\}$ is an $\alpha^{*}$-set as $\operatorname{int}(\operatorname{cl}(\operatorname{int}(A)))=\emptyset=\operatorname{int}(A)$. But $A$ is not a $P S$-regular set because $i_{\sigma}\left(c_{\sigma}(A)\right)=[A \cap \operatorname{cl}(\operatorname{int}(\operatorname{cl}(A))] \cup \operatorname{int}(\operatorname{cl}(A))=$ $[\{a, b\} \cap \operatorname{cl}(\{b, c\})] \cup\{b, c\}=\{a, b\} \cup\{b, c\}=\{a, b, c\} \neq A$.

Corollary 32. Every PS-regular set is a B-set, C-set, and $D(C, \alpha)$-set.

Proof. It is because every PS-regular set is $t$-set and $\alpha^{*}$-set.

Now, we provide some decompositions of regular closed sets using $P S$-regular sets. 
Theorem 33. For a topological space, the following are equivalent:

(i) A is regular closed;

(ii) $A$ is closed and PS-regular;

(iii) $A$ is $\alpha$-closed and PS-regular;

(iv) $A$ is preclosed and PS-regular.

Proof. (i) $\Rightarrow$ (ii): they are proved in Theorem 16 .

(ii) $\Rightarrow$ (iii): let $A$ be a closed set; then it is $\alpha$-closed because every closed set is $\alpha$-closed.

(iii) $\Rightarrow$ (iv): they are obvious because every $\alpha$-closed set is preclosed as well.

(iv) $\Rightarrow$ (i): let $A$ be a preclosed and $P S$-regular set. Then $A$ is semiopen. Thus $A \subseteq \operatorname{cl}(\operatorname{int}(A))$. Since $A$ is preclosed, therefore $\operatorname{cl}(\operatorname{int}(A)) \subseteq A$ as well. Hence $A=\operatorname{cl}(\operatorname{int}(A))$ and is hence regular closed.

Now, we proceed to provide decompositions of regular open sets using $P S$-regular sets.

Theorem 34. For a topological space, the following are equivalent:

(i) $A$ is regular open;

(ii) $A$ is open and PS-regular;

(iii) $A$ is preopen and PS-regular;

(iv) $A$ is preopen and semiclosed.

Proof. (i) $\Rightarrow$ (ii): they are proved earlier in Theorem 14 .

(ii) $\Rightarrow$ (iii): let $A \subseteq X$ be an open set. Then it is preopen because every open set is preopen.

(iii) $\Rightarrow$ (iv): as every PS-regular set is semiclosed therefore $A$ is semiclosed.

(iv) $\Rightarrow$ (i): let $A$ be a preopen and semiclosed set. Thus $A \subseteq \operatorname{int}(\operatorname{cl}(A))$ and $c_{\sigma}(A)=A$; that is, $\operatorname{int}(\operatorname{cl}(A)) \subseteq A$. Hence $A=\operatorname{int}(\operatorname{cl}(A)$. Thus $A$ is regular open.

Theorem 35. A set $A \subseteq X$ is regular open if and only if it is $\alpha$-open and PS-regular.

Proof. We have already proved in Theorem 34 that every regular open set is PS-regular and open and hence $\alpha$-open. Conversely, let $A$ be $P S$-regular; then it is an $\alpha^{*}$-set by Theorem 29. Hence $A$ is regular open because a set is regular open if and only if it is $\alpha$-open and $\alpha^{*}$-set [10].

\section{5. $P S$-Continuity and Almost $P S$-Continuity}

We first recall the following definitions.

Definition 36. A function $f: X \rightarrow Y$ is said to be $R$-map [18] (resp., almost continuous [19], almost $\alpha$-continuous [20], almost semicontinuous [21], and almost precontinuous [18]) if $f^{-1}(V)$ is regular open, (resp., open, $\alpha$-set, semiopen, and preopen) for every regular open set $V$ in $Y$.
Now we define PS-continuity and almost PS-continuity in the following way.

Definition 37. (a) A mapping $f: X \rightarrow Y$ is said to be PScontinuous at a point $x \in X$ if for every neighbourhood $M$ of $f(x)$ there exists a $P S$-regular neighbourhood $N$ of $x$ such that $f(N) \subseteq M$.

(b) A mapping $f: X \rightarrow Y$ is said to be almost PScontinuous at a point $x \in X$ if for every neighbourhood $M$ of $f(x)$ there exists a $P S$-regular neighbourhood $N$ of $x$ such that $f(N) \subseteq \operatorname{int} \operatorname{cl}(M)$.

A mapping $f: X \rightarrow Y$ is said to be PS-continuous (resp., almost PS-continuous) if it is $P S$-continuous (resp., almost $P S$-continuous) at each point $x$ of $X$.

Since every regular open set is open, therefore every PScontinuous mapping is almost $P S$-continuous.

Theorem 38. For a mapping $f: X \rightarrow Y$, the following are equivalent:

(i) $f$ is PS-continuous;

(ii) inverse image of every open subset of $Y$ is PS-regular;

(iii) inverse image of every closed subset of $Y$ is PS-regular.

Proof. (i) $\Rightarrow$ (ii): let $U$ be any open subset of $Y$ and let $x \in$ $f^{-1}(U)$. Then $f(x) \in U$. Therefore there exists a PS-regular subset $V$ in $X$ such that $x \in V$ and $f(V) \subseteq U$. Thus $x \in V \subseteq$ $f^{-1}(U)$; therefore $f^{-1}(U)$ is a $P S$-regular neighbourhood of $x$. Hence $f^{-1}(U)$ is $P S$-regular.

(ii) $\Rightarrow$ (iii): let $A$ be any closed subset of $Y$. Then $Y \backslash A$ is open and therefore $f^{-1}(Y \backslash A)$ is $P S$-regular; that is, $X \backslash f^{-1}(A)$ is $P S$-regular. Hence $f^{-1}(A)$ is $P S$-regular.

(iii) $\Rightarrow$ (i): let $M$ be open neighbourhood of $f(x)$; therefore $Y \backslash M$ is closed, and consequently $f^{-1}(Y \backslash M)$ is $P S$-regular. Thus $f^{-1}(M)$ is also $P S$-regular and hence $x \in$ $f^{-1}(M)=N$ (say). Then $N$ is a $P S$-regular neighbourhood of $x$ such that $f(N) \subseteq M$.

Theorem 39. For a mapping $f: X \rightarrow Y$, the following are equivalent:

(i) $f$ is almost PS-continuous at $x \in X$;

(ii) for every regular open neighbourhood $M$ of $f(x)$, there is a PS-regular neighbourhood $N$ of $x$ such that $f(N) \subseteq$ $M$.

Proof. (i) $\Rightarrow$ (ii): if $f$ is almost PS-continuous at $x$ and $M$ is a regular open neighbourhood of $f(x)$, then there is a PSregular neighbourhood $N$ of $x$ such that $f(N) \subseteq$ int $\operatorname{cl}(M)=$ M.

(ii) $\Rightarrow$ (i): it is obvious.

Theorem 40. For a mapping $f: X \rightarrow Y$, the following are equivalent:

(i) $f$ is almost PS-continuous;

(ii) inverse image of every regular open subset of $Y$ is PSregular; 
(iii) inverse image of every regular closed subset of $Y$ is PSregular;

(iv) for each point $x$ of $X$ and for each regular open neighbourhood $M$ of $f(x)$, there is a PS-regular neighbourhood $N$ of $x$ such that $f(N) \subseteq M$.

Proof. The proof is the same as Theorem 38.

Theorem 41. If $f: X \rightarrow Y$ is a PS-continuous (resp., almost PS-continuous) map then it is semicontinuous (resp., almost semicontinuous).

Proof. Let $A$ be any open (resp., regular open) subset in $Y$. Then $f^{-1}(A)$ is a $P S$-regular and hence a semiopen set because $f$ is $P S$-continuous (resp., almost $P S$-continuous). Hence $f$ is semicontinuous (resp., almost semicontinuous).

Theorem 42. If $f: X \rightarrow Y$ is an almost PS-continuous map then the following hold:

(i) $f$ is R-map if and only if it is almost precontinuous;

(ii) $f$ is $R$-map if and only if it is almost $\alpha$-continuous.

Proof. (i) Let $f: X \rightarrow Y$ be a $R$-map and let $U$ be any regular open subset in $Y$. Then $f^{-1}(U)$ is regular open and hence preopen. Thus $f$ is almost precontinuous.

Conversely, let $f$ be almost precontinuous and almost $P S$ regular continuous map. Let $U$ be any regular open subset of $Y$. Then $f^{-1}(U)$ is preopen as well as $P S$-regular. Hence $f^{-1}(U)$ is regular open. Therefore $f$ is a $R$-map.

(ii) It is the same as (i).

Remark 43. From the above theorem we can conclude that a map is almost continuous (a.c.s) if it is almost precontinuous and almost $P S$-continuous.

Theorem 44. Let $f:(X, \tau) \rightarrow(Y, \sigma)$ be a function and let $g:(X, \tau) \rightarrow(X \times Y, \tau \times \sigma)$ be the graph function defined by $g(x)=(x, f(x))$, for every $x \in X$. Then $f$ is almost PScontinuous if $g$ is almost PS-continuous.

Proof. Let $x \in X$ and $V \in R O(Y, \sigma)$ containing $f(x)$. Then, we have $g(x)=(x, f(x)) \in X \times Y \in R O(X \times Y, \tau \times \sigma)$. Since $g$ is almost $P S$-continuous, there exists a $P S$-regular set $U$ of $X$ containing $x$ such that $g(U) \subseteq X \times Y$. Therefore we obtain $f(U) \subseteq V$ and hence $f$ is almost $P S$-continuous.

\section{Conflict of Interests}

The authors declare that there is no conflict of interests regarding the publication of this paper.

\section{Acknowledgments}

The authors express sincere thanks to the referees of the paper. The suggestions provided by one of the referees have greatly improved the presentation of the paper. This work is a part of a research work financed by the University Grant Commission (India).

\section{References}

[1] E. Fabrizi and A. Saffiotti, "Behavioural navigation on topologybased maps," in Proceedings of the 8th Symposium on Robotics with Applications, Maui, Hawaii, USA, 2000.

[2] V. Kovalesky and R. Kopperman, "Some topology-based image processing algorithms," Annals of the New York Academy of Sciences, vol. 728, pp. 174-182, 1994.

[3] B. M. R. Stadler and P. F. Stadler, "Generalized topological spaces in evolutionary theory and combinatorial chemistry," Journal of Chemical Information and Computer Sciences, vol. 42, no. 3, pp. 577-585, 2002.

[4] Á. Császár, “Generalized open sets," Acta Mathematica Hungarica, vol. 75, no. 1-2, pp. 65-87, 1997.

[5] Á. Császár, "On the $\gamma$-interior and $\gamma$-closure of a set," Acta Mathematica Hungarica, vol. 80, no. 1-2, pp. 89-93, 1998.

[6] Á. Császár, “Generalized topology, generalized continuity, Acta Mathematica Hungarica, vol. 96, no. 4, pp. 351-357, 2002.

[7] Á. Császár, "Generalized open sets in generalized topologies," Acta Mathematica Hungarica, vol. 106, no. 1-2, pp. 53-66, 2005.

[8] D. Peleg, "A generalized closure and complement phenomenon," Discrete Mathematics, vol. 50, no. 2-3, pp. 285-293, 1984.

[9] B. A. Davey and H. A. Priestley, Introduction to Lattices and Order, Cambridge University Press, 2nd edition, 2003.

[10] E. Hatir, T. Noiri, and S. Yüksel, "A decomposition of continuity," Acta Mathematica Hungarica, vol. 70, no. 1-2, pp. 145-150, 1996.

[11] J. Dugundji, Topology, Allyn and Bacon, Boston, Mass, USA, 1972.

[12] V. Popa and T. Noiri, "On the definitions of some generalized forms of continuity under minimal conditions," Memoirs of Faculty of Science, Kochi University A: Mathematics, vol. 22, pp. 31-41, 2001.

[13] R. Jamunarani and P. Jeyanthi, "Regular sets in generalized topological spaces," Acta Mathematica Hungarica, vol. 135, no. 4, pp. 342-349, 2012.

[14] R. D. Sarma and A. Gupta, "On $\pi$ gs- $\lambda$ closed sets in generalized topological spaces," in Proceedings of the 21st International Conference of FIM on Interdisciplinary Mathematics, Statistics and Computational Techniques, Chandigarh, India, 2012.

[15] C. Dorsett, "Semi-connectedness," Indian Journal of Mechanics and Mathematics, vol. 717, no. 1, pp. 57-63, 1979.

[16] J. Tong, "A decomposition of continuity in topological spaces," Acta Mathematica Hungarica, vol. 54, pp. 51-55, 1989.

[17] M. Przemski, "A decomposition of continuity and $\alpha$-continuity," Acta Mathematica Hungarica, vol. 61, no. 1-2, pp. 93-98, 1993.

[18] A. A. Nasef and T. Noiri, "Some weak forms of almost continuity," Acta Mathematica Hungarica, vol. 74, no. 3, pp. 211-219, 1997.

[19] M. K. Singal and A. R. Singal, "Almost-continuous mappings," Yokohama Mathematical Journal, vol. 2, pp. 63-73, 1968.

[20] T. Noiri, "Almost $\alpha$-continuous functions," Kyungpook Mathematical Journal, vol. 28, no. 1, pp. 71-77, 1988.

[21] A. S. Mashhour, M. E. Abd EI-Monsef, and S. N. El-Deeb, "On ore-continuous and weak pre-continuous mappings," in Proceedings of the Mathematical and Physical Society of Egypt, vol. 51, 1981. 


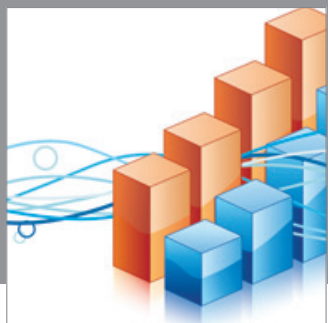

Advances in

Operations Research

mansans

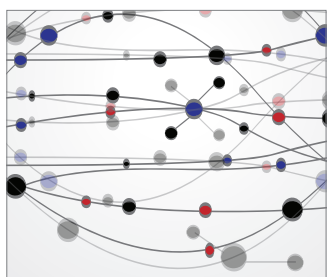

The Scientific World Journal
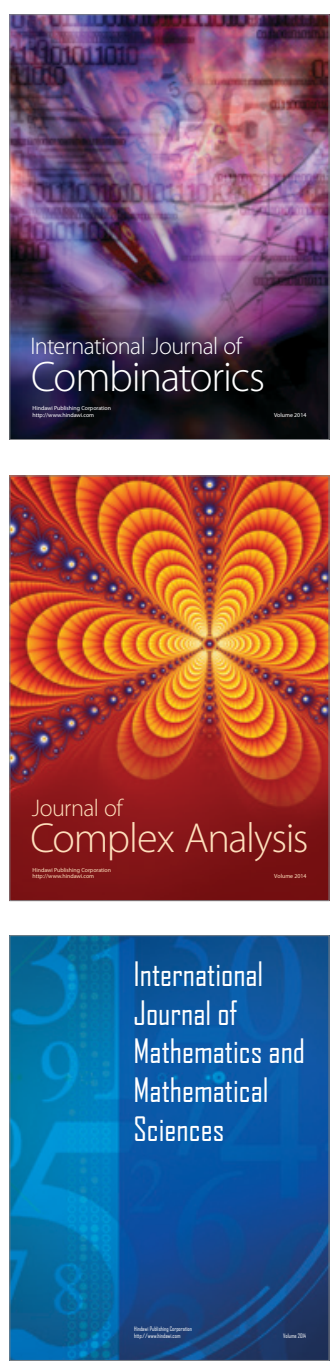
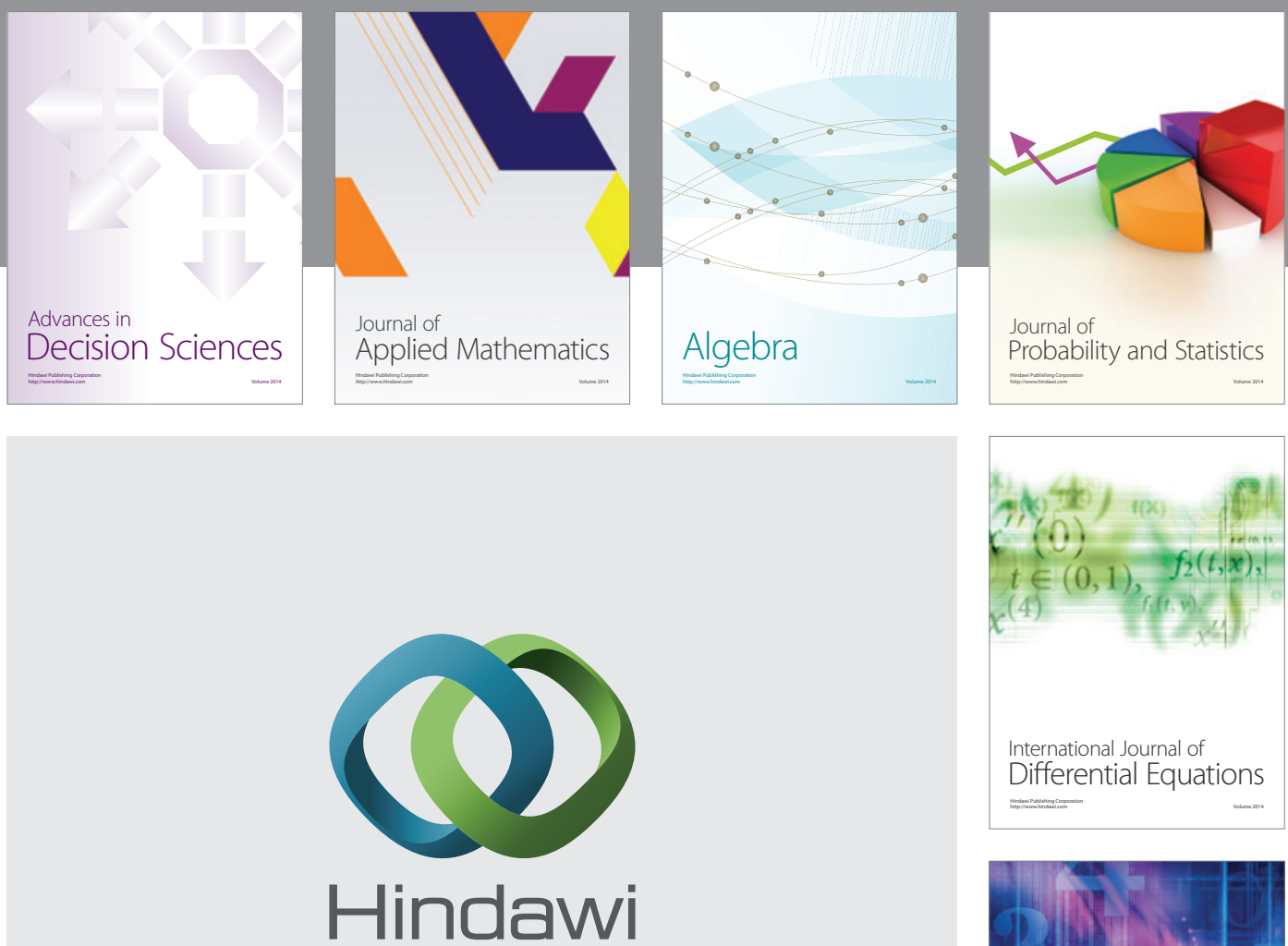

Submit your manuscripts at http://www.hindawi.com
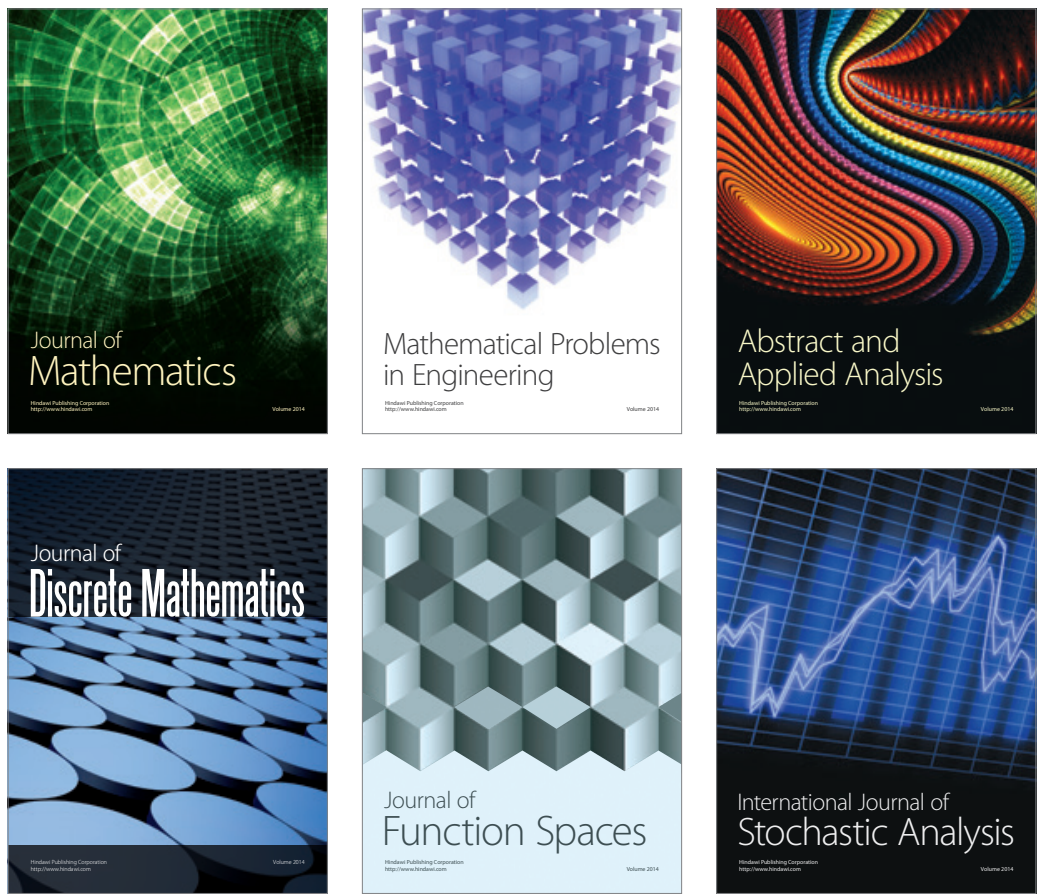

Journal of

Function Spaces

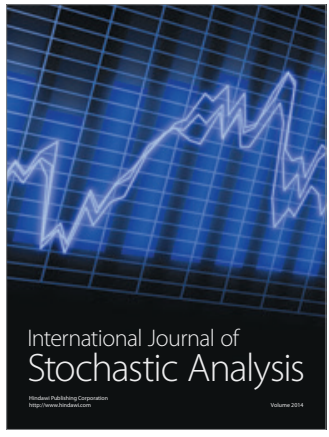

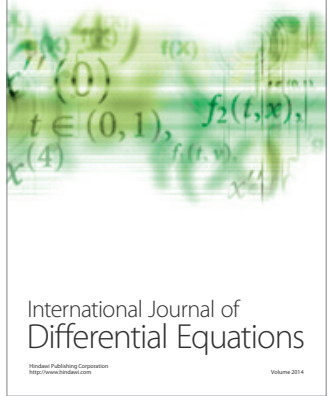
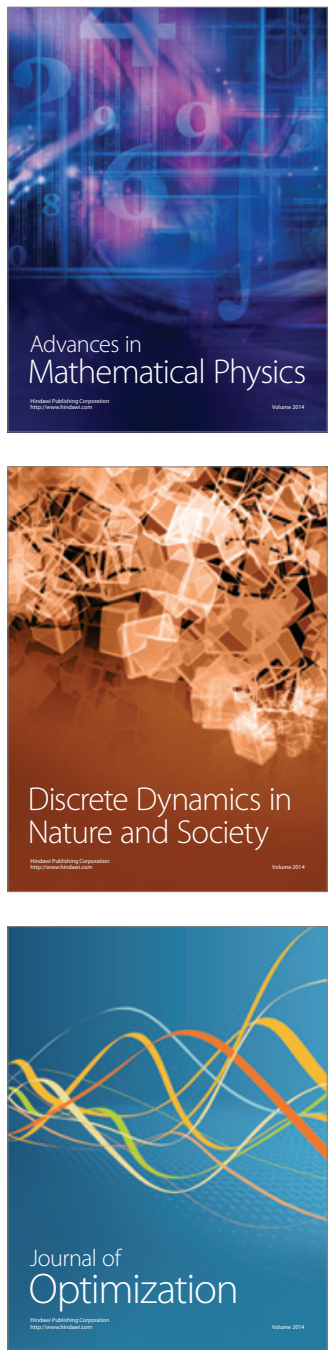